\title{
Optimización Matemática como Herramienta para la Toma de Decisiones en la Empresa
}

Mathematical Optimization as a Tool for Decision-Making in the Company

Villarreal F., ${ }^{1}$ Montenegro D.,2 Nuñez J. 3
1 Universidad de Los Hemisferios, Facultad de Ciencias Económicas y Empresariales, Quito-Ecuador
1 Universidad de Girona, Facultad de Ciencias Económicas y Empresariales, Comunidad de Cataluña-España
e-mail: leninv@ uhemisferios.edu.ec
2 IDE Business School, Quito-Ecuador
2Universidad de Los Hemisferios, Quito-Ecuador
e-mail: diegom@ @hemisferios.edu.ec
e-mail: janneth.nunez@emgirs.gob.ec
3 Empresa Pública Metropolitana de Gestión Integral y Residuos Sólidos, Quito-Ecuador
Decibido: 5/01/2021
Aceptado: 14/04/2021

\begin{abstract}
RESUMEN
El presente trabajo de investigación se basa en una propuesta de generación de un modelo de programación lineal basado en la metodología simplex, cuyo objetivo es generar la optimización de recursos para las empresas respecto a la selección de medios publicitarios con un presupuesto limitado y fijo, que está sujeto a un costo de medios y posibles coberturas a potenciales clientes como valor esperado. El modelo persigue maximizar la audiencia como agentes de generación de valor para el crecimiento de las instituciones, teniendo en cuenta la conformación de un portafolio de opciones que les permitan a las empresas capitalizar esta cobertura, ser conocidas y ganar reputación en el mercado, con el objetivo final de rentabilizar la inversión. Se realiza una revisión de conceptos relacionados a la programación lineal y aplicación del algoritmo simplex matricial, como método matemático de aporte de información técnica, para una adecuada toma de decisiones de la empresa, cuyo principal resultado es la asignación de publicidad en diferentes medios como televisión, radio y redes sociales; siendo este último uno de los medios más accesibles y efectivos para que las empresas y sus marcas sean reconocidas. Se pueden realizar varias simulaciones y adecuaciones según convenga.
\end{abstract}

Palabras clave: Optimización matemática; método simplex; maximizar audiencia; medios publicitarios.

\begin{abstract}
This research work is based on a proposal for the generation of a linear programming model based on the simplex method methodology, whose objective is to generate the optimization of resources for companies regarding the selection of advertising media with a limited and fixed budget, which is subject to a cost of means and possible coverage to potential clients as expected value. The model seeks to maximize the audience as agents of value generation for the growth of the institutions, taking into account the formation of a portfolio of options that allow companies to capitalize on this coverage, be known and gain a reputation in the market, with the final goal of making the investment profitable. A review of concepts related to linear programming and application of the simplex matrix algorithm is carried out, as a mathematical method of providing technical information, for an adequate decision-making of the company, whose main result is the allocation of advertising in different media such as television, radio and social networks; the latter being one of the most accessible and effective means for companies and their brands to be recognized. Various simulations and adjustments can be made as appropriate.
\end{abstract}

Keywords: Mathematical optimization; simplex method; maximize audience; advertising media. 


\section{Introducción}

Las primeras actividades formales de la investigación de operaciones se dan en Inglaterra durante la Segunda Guerra Mundial con motivo de la logística del material bélico que debía transportarse y optimizar tiempo y recursos para llegar al destino adecuado lo más pronto posible. Los mandos militares solicitaron la colaboración de científicos para la resolución en tiempo real de problemas estratégicos y tácticos en el desarrollo de la guerra. De ahí en adelante, esta técnica se fue depurando y ambientándose a los esquemas empresariales para mejorar la eficiencia en la gestión administrativa, financiera, operativa, logística y toda actividad que conlleve a mejorar la productividad de los agentes económicos [1].

Varios de los avances de la programación matemática se deben a que en la actualidad se encuentran asociados al desarrollo y uso de tecnologías como las aplicaciones de software para generar y analizar mega datos, métodos cada vez más abreviados de cálculo matemático, que hacen posible que aquellos problemas que tardaban días, semanas, meses incluso hasta años, se puedan resolver casi de manera inmediata.

La investigación operativa es un proceso de mejora continua, pues con el pasar de los años, se encuentran varias utilidades a sus aplicaciones, como, por ejemplo, en la década de los setenta surge con Farrell una medida satisfactoria de eficiencia productiva, que toma en cuenta todos los inputs o recursos empleados generado una salida llamada output que es el resultado de la eficiencia [2]. Es importante indicar que esta rama de las matemáticas reúne un conjunto de aplicaciones en varios campos como la Física, Economía, Sociología y en especial en la Administración de Empresas, debido a que procura encontrar la una relación causa-efecto mediante la aplicación de un modelo matemático. En el ámbito empresarial es muy común la aplicación de modelos de optimización para la selección de medios de comunicación, y en publicidad para generar la mezcla adecuada de medios a ser seleccionados, de manera que, partiendo de la asignación de un presupuesto fijo como recurso limitado, su respuesta permita abarcar la mayor audiencia posible [2].
En materia de investigación de mercados se puede aplicar la investigación operativa en el levantamiento de encuestas [3], en programación de la producción se utiliza para establecer un programa donde se toma en cuenta el tiempo para maximizar o minimizar la ganancia o el costo respectivamente, en la planeación de la mano de obra es usada para calcular las necesidades de personal durante cierto período, donde, por ejemplo, los gerentes asignan individuos a puestos de trabajo que requieren talentos afines y flexibles a la temporada [3]. De igual forma, la investigación operativa sirve para la selección de portafolios de inversiones entre varias alternativas, y así maximizar el rendimiento de la empresa o de clientes en las instituciones financieras, tomando en cuenta el riesgo y las restricciones legales [4]. En marketing para medir la efectividad de una campaña publicitaria, considerando que la optimización de los recursos es clave a la hora de acaparar más audiencia, puesto que a mayor número de personas que llegue un mensaje, este puede convertirse en posibles futuras ventas y referencias en el mercado de consumo de un bien o servicio. Las aplicaciones de la programación lineal se muestran a continuación:

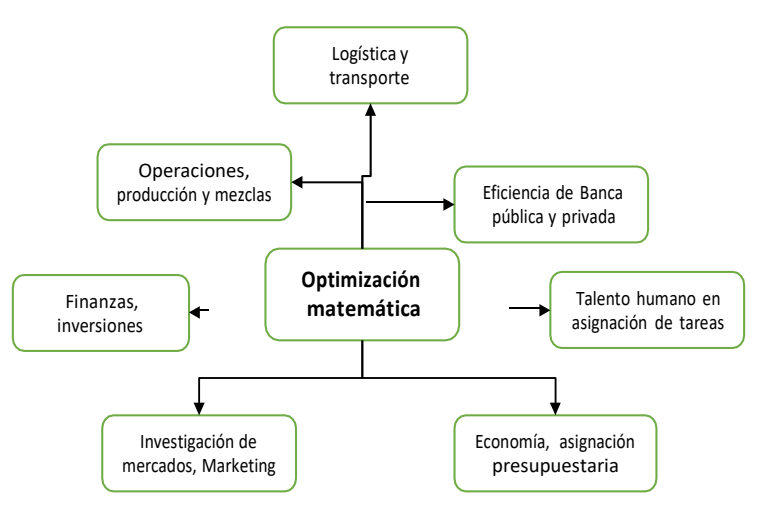

Figura 1. Aplicaciones a la programación lineal Fuente: Elaborado por los autores a partir de (Coll y Olga 2006).

Esta medición se realiza a través de plataformas para obtener una visión holística e integrada de cada campaña publicitaria, que a su vez está respaldada con calidad y precisión para intentar 
convertir a la empresa en líder de la industria. Las personas de la sociedad contemporánea viven en una red de «medios» que transmiten y hacen posible las noticias, la información y la publicidad. Existen muchos conceptos utilizados en la programación de medios como: la compra, alcance, frecuencia, impresiones, participantes clave y representantes de medios, lo que hace compleja su administración [5]; además, esta actividad tiene como insumos y productos a anunciantes, agencias, televisión, radio, internet, plataformas en redes sociales, dispositivos móviles y tabletas. El resultado final de la mezcla de todas estas premisas es la realización de una campaña de comunicación en tiempo real.

\section{Revisión bibliográfica}

Bermúdez [6] genera un estudio documental informativo, en el que aborda los aportes de la comunidad científica, respecto a temas relacionados con programación lineal, en las que evidencia que los modelos de optimización basados en programación lineal son utilizados en ambientes reales para la solución de problemas y ayuda a la toma de decisiones que contribuyen a la reducción de costos, mejorar la productividad en la planificación de la producción para el aprovechamiento de la capacidad de reducción en inventarios.

Por otro lado, Alvarado [7] hace una revisión de la aplicación en la programación lineal en la toma de decisiones en la gestión de la pymes (pequeñas y medianas empresas) como base de eficiencia en el desarrollo social, en el que recomienda que los profesionales de esta industria obtengan una mayor habilidad en el manejo de esta técnica. Bona et. al [8] hablan en su estudio sobre el desarrollo en la optimización de procesos con variables continuas y variables cualitativas continuas, a partir de la metodología simplex. En el software generado, realizan modelos predictivos en dos procesos distintos.

Existen aspectos relevantes de la experimentación numérica en el algoritmo PSO, o conocido como optimización de enjambre de partículas, de un algoritmo híbrido hacia el método simplex que optimiza la iteración de los parámetros con una topología que permite generar este cambio con el método simplex modificado [9]. Para complemen- tar, De la Hoz, Vélez y López [10] presentan un modelo de programación lineal multi - objetivo para logística inversa del polipropileno, donde los principales resultados muestran un mejoramiento del $12,6 \%$ en los costos asociados al programa de planeación en los procesos asociados de recuperación del plástico, proveniente de los residuos industriales sólidos urbanos con la función de minimización de costos.

Noguera y Posada [11] generan un estudio de programación lineal aplicado a las raciones para rumiantes (vacas lecheras) en el que estiman un balance adecuado en la fabricación de alimentos concentrados al menor costo posible, es decir, un problema de programación lineal cuya función objetivo es minimizar el costo del alimento con la herramienta de Solver de Excel para Windows; concluyendo que desde el punto de vista académico la formulación de «dietas» utilizando programación lineal, permite crear distintos escenarios productivos para que el investigador tenga mayor criterio para la toma de decisiones.

En operaciones, los modelos de programación lineal son útiles para la planificación de la producción en que, utilizando una función objetivo de minimización de costes en cuanto al tiempo y los recursos y la consideración del nivel mínimo de servicio ligado a la demanda diferida, los resultados exponen una mejora en el modelo propuesto respecto al procedimiento actual del estudio [12]. De la misma forma, Ortiz y Caicedo [13]. diseñan un procedimiento para la programación y control de la producción de una empresa de calzado con programación lineal y la teoría de restricciones. El modelo identificó las cantidades óptimas de fabricación, donde la función objetivo es maximizar el throughput, procedimiento que permitió controlar de manera eficiente la producción de calzado con un modelo que a su vez puede ser emulado por empresas de condiciones y negocio similares.

En el caso de manejo y gestión de proyectos, la investigación de operaciones es útil como lo señala Terrazas [14] para la mejor asignación de recursos en actividades competidoras. En el análisis de factibilidad de proyectos una dificultad es saber ubicar un proyecto en las mejores condiciones 
operativas para minimizar costos y maximizar ganancias. El beneficio radica en la resolución de problemas técnicos de localización para encontrar la viabilidad económica.

En la rama de las finanzas, la optimización matemática ha incursionado de manera efectiva para la toma de decisiones. María [15] aplica un modelo de optimización lineal en el portafolio de diecisiete proyectos agroindustriales con restricción de inversión para maximizar el retorno al inversor, permitiendo que el modelo trabaje en la combinación de variables, considerando la tasa de oportunidad para el stakeholder. Prieto, González y Arce [16] generan un modelo de optimización de selección de cartera para relacionar la rentabilidad esperada ligada a la esperanza matemática y el riesgo inherente de la cartera dado por la desviación estándar. En su estudio de caso evidencian un aumento de la tasa de rendimiento de la cartera en cinco activos analizados durante diez períodos de tiempo, basados en las teorías definidas por Harry Markowitz.

Producir y vender frente a gestionar clientes ha sido el dilema de marketing (y de la publicidad) que no se ha podido resolver hasta ahora. La ciencia del management hay que abordarla como un corpus doctrinal al servicio del éxito en los negocios y es clave aprovechar las oportunidades del mercado para obtener una rentabilidad mejor [17]. Schumpeter [18], el menos interesado de todos los gurús por el beneficio, pone en manos de los productores la capacidad de impulsar el crecimiento económico de la sociedad y enfatiza el valor social del emprendimiento. Chandler [19] parte del análisis de los casos para concebir la estrategia como el camino al éxito. Porter [20] se adentra en el análisis de las fuerzas competitivas del mercado para alcanzar aquellas ventajas que conducen a una mejor rentabilidad de la inversión. Mintzberg [21] menciona que la estrategia ha de definirse a través de la integración y la complementariedad de sus distintas acepciones: como plan o guía para abordar una situación, como pauta o acción para ganar a la competencia, como patrón o comportamiento que se quiere producir, como posición y perspectiva de percibir las cosas.
Y dentro de esta perspectiva se encuentra el marketing, mercadotecnia, mercadeo, estrategia comercial, publicidad, propaganda, gestión de mercado, etc. Todas estas palabras se usan para abarcar todo lo que incumbe a las relaciones entre las empresas y los clientes. Originalmente, el marketing se responsabilizaba de vender a los clientes los productos fabricados por los estrategas; hasta bastante más tarde, el marketing es concebido como el conjunto de actividades empresariales que dirigen el flujo de bienes y servicios del productor al consumidor. En las revisiones posteriores del concepto por parte de la AMA (American Marketing Association), surge la idea de intercambio y adquiere relevancia la creación de valor: el marketing es la actividad, el conjunto de instituciones y procesos para crear, comunicar, distribuir e intercambiar ofertas que tengan valor para los consumidores, los clientes, los socios y la sociedad en general [22]. Montenegro y Calvache [23] van más allá al mencionar que el marketing tradicional se ha olvidado de los detalles para descubrir pensamientos ocultos (creencias) y sensaciones motivantes; «el marketing de ahora en adelante debe dejar de lado las teorías tradicionales y atreverse a generar o descubrir otras con una predisposición disruptiva, arremetiendo técnicamente hacia una nueva forma de ver y entender al cliente, valorando sus sentimientos y sensaciones».

Como parte fundamental de este marketing moderno está la comunicación. La comunicación es un conjunto de elementos y actividades dedicados a la interacción de mensajes, productos, marcas, ideas e informaciones entre la organización, el cliente y los stakeholders que, de la manera más eficaz posible, se convierte en flujos permanentes de intercambios. En el marketing tradicional, la relación era unilateral: el emisor era la empresa y los receptores los clientes a los cuales había que buscar y presionar para que aceptaran el mensaje y los soportes, los medios de comunicación se gestionaban mayoritariamente desde los emisores. Ahora, todos los agentes son emisores y receptores, al tiempo que actúan codificando y descodificando los contenidos que surgen en medio de los importantes ruidos que se generan a lo largo del proceso de transmisión; por su parte, internet ha desbloqueado la unidireccionalidad al otorgar poder para emitir mensajes a su antojo [17]. 
Para esto, la publicidad usa los medios de comunicación masiva como parte esencial de sus mensajes, para llevar información persuasiva a los mercados de la empresa. Mientras una pieza no se difunda, no existe mensaje. Cuando se piensa algo o se tiene un arte final en la pantalla del computador, no se ha generado todavía comunicación: hay que establecer contacto con el receptor. Sin medios no existe publicidad. Es tal la importancia de los medios, que a medida que estos cambian, la publicidad evoluciona [24]. La transformación digital ha llevado a la publicidad a los medios en línea. Para Wells, Moriarty y Burnett [5] este tipo de comunicación tiene como propósito dar un mensaje de recordación de marca a quienes visitan una página web (o redes sociales), transmite un mensaje informativo y persuasivo; sin embargo, el más importante de los propósitos es la compra instantánea o la viralización de la oferta. Por otra parte, los medios de comunicación masiva utilizan su capacidad de llegar a las personas, vender publicidad, difundir ideas políticas, posicionar marcas, por su gran capacidad de influencia sobre los núcleos de población a los que van dirigidos. Una buena estrategia publicitaria muy probablemente generará beneficios extraordinarios a las firmas que sepan aprovecharlas. Pero, cuando esta capacidad de influencia sobre la población se utiliza inadecuadamente puede generar injusticias para quienes no pueden acceder a dichos medios masivos.

En las aplicaciones de las tácticas del marketing, será muy usual optimizar los recursos asignados a la selección de medios de comunicación, que ayudan a los directivos y gerentes a repartir recursos fijos para extractos o comerciales en periódicos, radio, revistas especializadas, canales de televisión, redes sociales y bases de datos de correos electrónicos. Para Anderson et. al [25] el objetivo del problema de programación lineal es maximizar la cobertura de la audiencia, basado en la frecuencia de repetición de la publicidad y la calidad de la información que se emite, y las restricciones están sujetas a políticas de la empresa, contratos y disponibilidad de los medios publicitarios.

\section{Metodología}

\section{El modelo de programación lineal}

Es importante el planteamiento de las restricciones que se van a utilizar, alternativas de decisión y la selección de criterios de la función del objetivo a maximizar o minimizar. Para Taha [26] la solución al modelo matemático responde a si satisface todas las restricciones y a su vez se optimiza cuando genera el mejor valor posible. La programación lineal involucra modelos que parten de la función objetivo y restricciones lineales; es decir, la variable se encuentra elevada a la potencia uno. Otros métodos son la programación entera en el que algunas variables asumen números enteros, así como también la programación dinámica que involucra al modelo original que se descompone en varios problemas pequeños pero manejables, la programación en red y la programación no lineal cuyas funciones son no lineales.

Las iteraciones son repeticiones que se encuentran dentro de los algoritmos que conducen a la solución óptima; pero, no todos los modelos matemáticos pueden ser resueltos con algoritmos, por lo que es necesario acudir a la solución heurística y meta-heurística.

\section{Fases del estudio de programación matemática}

Las fases que conducen a la resolución del modelo de programación lineal (Figura 2), parte de la definición del problema, generación de base de datos y variables, desarrollo de iteraciones hasta llegar a la solución óptima principalmente. Este tipo de modelos, tal como ocurre en proyectos tecnológicos de desarrollo de aplicaciones, debe trabajarse en sociedad, donde los expertos en la modelación matemática trabajan en conjunto con quiénes demandan la solución a un problema; considerándose así a la programación matemática como una ciencia y un arte [3]. Soot [27] afirma que sin importar el campo del cual provenga el problema y lo específico del tema abordado, se analiza el proceso con un modelo matemático que contiene las fases de la programación matemática, tal como se indica en la siguiente figura: 


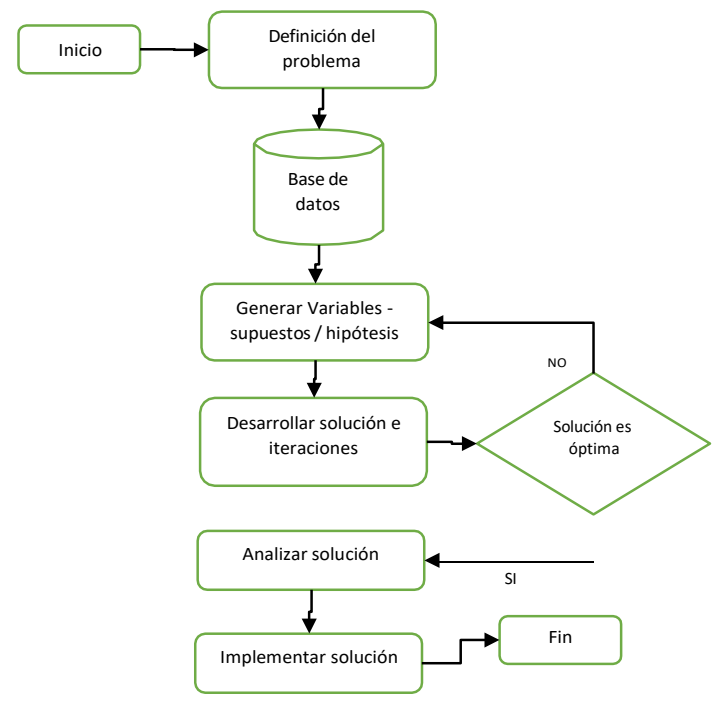

Figura 2. Fases del proceso de modelado de programación matemática

Render, Stair y Hanna [3] muestran varias propiedades a tomar en cuenta en los problemas de programación matemática, estas son:

- Los problemas de programación matemática tienen como función objetivo maximizar o minimizar.
- Los problemas de programación matemática disponen de una o más restricciones.

- Siguen cursos de acción alternativos.

- Poseen certeza y divisibilidad.

- Las variables son no negativas.

\section{El método Simplex}

Es un procedimiento general de programación matemática para llegar a la solución óptima que se obtiene en un número finito de pasos. Estos son:

- La solución es cualquier conjunto de variables que satisfacen las restricciones del problema $(\mathrm{Ax}=\mathrm{B})$.

- La solución factible es aquella que satisface la no - negatividad de las restricciones $\left(X_{j \geq 0}\right)$.

- La solución básica del sistema de $m$ ecuaciones lineales son $n$ variables $A x=B,(m<n)$, cuyo rango $R(A)=m$ (variables básicas). La solución es $n-m=0 ; \mathrm{y}$, resolviendo para las $m$ variables restantes, cuando el determinante de sus coeficientes no sean cero.

- La solución básica factible es tal, que todas las variables básicas $x_{j \geq 0}$

- La degeneración de una solución básica se da si una o más variables básicas son iguales a cero.

\section{Procedimiento de forma matricial}

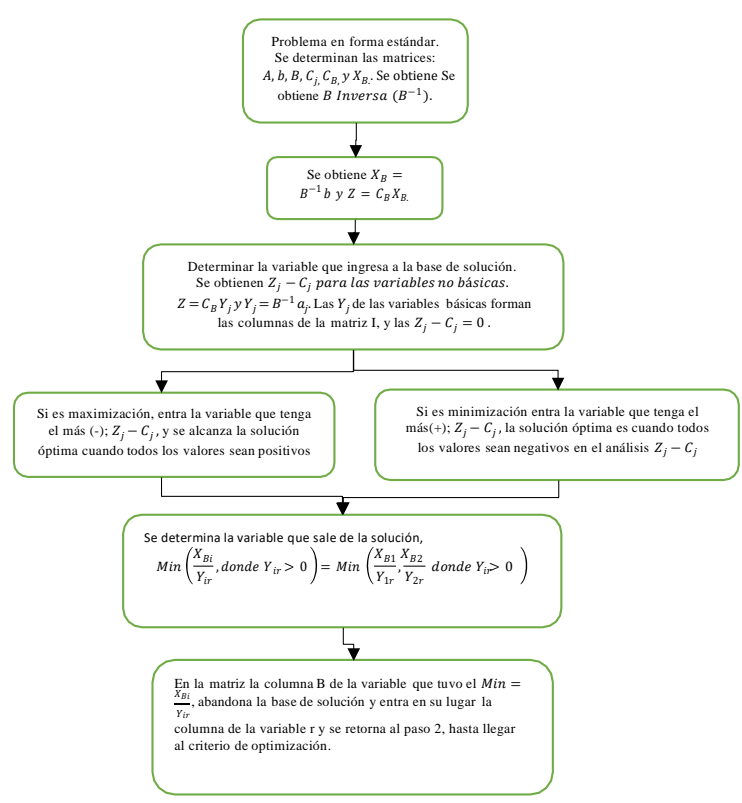

Figura 3. Proceso de metodología Simplex

Fuente: Notas del método simplex. Martínez, H. 


\section{Tabla del método Simplex}

Tabla1. Matriz Método Simplex.

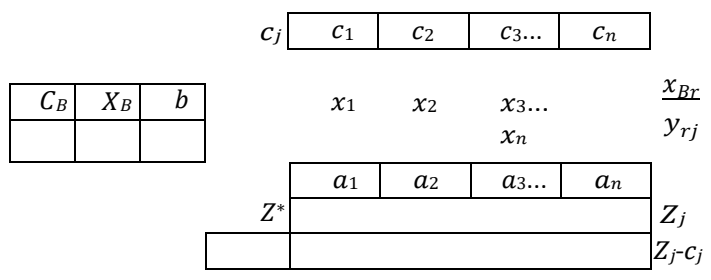

Fuente: Notas del método simplex. Martínez, H.

Donde:

$\mathrm{X}_{\mathrm{B}}=$ Vector de la solución básica factible. $b=$ variables básicas.

$Z^{*}=$ función objetivo (Max - Min)

$\mathrm{C}_{\mathrm{j}}=$ Vector de costos y $\mathrm{C}_{\mathrm{B}}$ Vector de componentes $\mathrm{C}$

$\mathrm{Z}_{\mathrm{j}}=\mathrm{CB}_{\mathrm{B}} \mathrm{X}_{\mathrm{B}}$ y $\mathrm{Z}_{\mathrm{j}} \mathrm{C}_{\mathrm{j}}=\mathrm{CB}_{\mathrm{B}} \mathrm{X}_{\mathrm{B}}-\mathrm{C}_{\mathrm{j}}$

$Y_{r j}=$ Nueva solución básica factible

Datos, definición de variables y modelo matemático

En la tabla siguiente, se recogen los datos e información de medios de comunicación y publicitarios para la investigación, tomados de fuente primaria de los costos proporcionados por los oferentes.

Tabla 2. Datos medios de comunicación, costos y audiencia.

\begin{tabular}{|c|c|c|c|c|c|c|c|}
\hline \multicolumn{8}{|c|}{ Televisión (semanal) } \\
\hline Canal & Horario & Variable & Programa & Costo & $\begin{array}{l}\text { Spot } \\
\text { (segundos) }\end{array}$ & Audiencia & $\begin{array}{l}\text { Máx. anuncios } \\
\text { por semana }\end{array}$ \\
\hline \multirow{3}{*}{ Ecuavisa } & Matutino & $\mathrm{x} 1$ & Contacto directo & $\$ 745,00$ & 30 & 178.750 & 9 \\
\hline & Vespertino & $\mathrm{x} 2$ & En contacto & $\$ 985,00$ & 30 & 121.960 & 10 \\
\hline & Nocturno & $\mathrm{x} 3$ & Noticiero & $\$ 1.150,00$ & 30 & 209.060 & 12 \\
\hline \multicolumn{8}{|c|}{ Radio } \\
\hline \multirow[b]{2}{*}{ Fluminense } & Matutino & $\mathrm{x} 4$ & Música & $\$ 360,00$ & 30 & 356.901 & 18 \\
\hline & $\begin{array}{l}\text { Vespertino } \\
\text { nocturno }\end{array}$ & $\mathrm{x} 5$ & Noticias & $\$ 315,00$ & 30 & 250.710 & 22 \\
\hline \multicolumn{8}{|c|}{ Prensa } \\
\hline Nombre & $\begin{array}{l}\text { Día de la } \\
\text { semana }\end{array}$ & Variable & $\begin{array}{l}\text { Ubicación } \\
\text { en portada }\end{array}$ & Costo & $\begin{array}{l}\text { I m p r e - } \\
\text { sión }\end{array}$ & Audiencia & $\begin{array}{l}\text { Máx. anuncios } \\
\text { por semana }\end{array}$ \\
\hline \multirow{2}{*}{ El Comercio } & $\begin{array}{l}\text { Lunes a } \\
\text { sábado }\end{array}$ & $x 6$ & Interior & $\$ 1.294,00$ & $\begin{array}{l}\text { Media } \\
\text { página }\end{array}$ & 284.762 & 5 \\
\hline & Domingo & $\mathrm{x} 7$ & Interior & $\$ 552,00$ & $\begin{array}{l}\text { Media } \\
\text { página }\end{array}$ & 466.728 & 1 \\
\hline \multicolumn{8}{|c|}{ Redes sociales } \\
\hline Nombre & $\begin{array}{l}\text { Día de la } \\
\text { semana }\end{array}$ & Variable & $\begin{array}{l}\text { Ubicación } \\
\text { en portada }\end{array}$ & Costo & $\begin{array}{l}\text { Imagen } \\
\text { Spot }\end{array}$ & Audiencia & $\begin{array}{l}\text { Máx. anuncios } \\
\text { por semana }\end{array}$ \\
\hline $\begin{array}{l}\text { Facebook } \\
\text { Instagram }\end{array}$ & Semana & $\mathrm{x} 8$ & Videos & $\$ 150,00$ & 5 & 50.000 & 45 \\
\hline
\end{tabular}

Fuente: Recolección de datos e información de medios publicitarios en Quito (2020). 


\section{Definición de variables}

x1=número de spots en Tv (1) de 30sg horario matutino

x2=número de spots en $\mathrm{Tv}$ (1) de 30sg horario vespertino

x3=número de spots en Tv (1) de 30sg horario nocturno

x4=número de spots en radio (1) de 30sg horario matutino

x $5=$ número de spots en radio (1) de 30sg horario vespertino/nocturno

x6=número de anuncios prensa (1) portada lunes a sábado

x7=número de anuncios prensa (1) interior/domingo

x8=número de spots en Facebook-Instagram /semana

\section{Modelo de programación matemática}

Generadas las variables, se escriben las expresiones matemáticas para el objetivo y las restricciones identificadas. Las restricciones de no negatividad se establecen en forma explícita.

Función objetivo: maximizar la cobertura de la audiencia con la mezcla de todas las variables, generando escenarios básicos.

Max $Z=17.8750 \times 1+121.960 \times 2+209.060 \times 3+356.90$ $1 \times 4+250.710 \times 5+284.762 \times 6+466.728 \times 7+50.000 \times 8$

\section{Restricciones (escenarios básicos):}

Como la restricción 1 es del tipo ' $\leq$ ' se agrega la variable de holgura X9.

Como la restricción 2 es del tipo ' $\leq$ ' se agrega la variable de holgura X10.

Como la restricción 3 es del tipo ' $\leq$ ' se agrega la variable de holgura X11.

Como la restricción 4 es del tipo ' $\leq$ ' se agrega la variable de holgura X12.

Como la restricción 5 es del tipo ' $\leq$ ' se agrega la variable de holgura X13.

Como la restricción 6 es del tipo ' $\leq$ ' se agrega la variable de holgura X14.

Como la restricción 7 es del tipo ' $\leq$ ' se agrega la variable de holgura X15.

Como la restricción 8 es del tipo ' $\leq$ ' se agrega la variable de holgura X16.

Como la restricción 9 es del tipo ' $\leq$ ' se agrega la variable de holgura X17.
Como la restricción 10 es del tipo ' $\leq$ ' se agrega la variable de holgura X18.

Como la restricción 11 es del tipo ' $\leq$ ' se agrega la variable de holgura X19.

\section{Modelo matemático de programación con la forma estándar:}

Se pasa el problema a la forma estándar, añadiendo variables de exceso, holgura, y artificiales según corresponda (mostrar/ocultar detalles)

MAXIMIZAR: $\mathrm{Z}=178750 \mathrm{X} 1+121960 \mathrm{X} 2+$ $209060 X 3+356901 X 4+250710 X 5+284762$ $\mathrm{X} 6+466728 \mathrm{X} 7+50000 \mathrm{X} 8$

\section{Sujeto a:}

$1 \times 1+0 \times 2+0 \times 3+0 \times 4+0 \times 5+0 \times 6+0 \times 7+0 \times 8 \leq 9$
$0 \times 1+2 \times 2+0 \times 3+0 \times 4+0 \times 5+0 \times 6+0 \times 7+0 \times 8 \leq 10$
$0 \times 1+0 \times 2+1 \times 3+0 \times 4+0 \times 5+0 \times 6+0 \times 7+0 \times 8 \leq 12$
$0 \times 1+0 \times 2+0 \times 3+1 \times 4+0 \times 5+0 \times 6+0 \times 7+0 \times 8 \leq 18$
$0 \times 1+0 \times 2+0 \times 3+0 \times 4+1 \times 5+0 \times 6+0 \times 7+0 \times 8 \leq 22$
$0 \times 1+0 \times 2+0 \times 3+0 \times 4+0 \times 5+1 \times 6+0 \times 7+0 \times 8 \leq 5$
$0 \times 1+0 \times 2+0 \times 3+0 \times 4+0 \times 5+0 \times 6+1 \times 7+0 \times 8 \leq 1$
$0 \times 1+0 \times 2+0 \times 3+0 \times 4+0 \times 5+0 \times 6+0 \times 7+1 \times 8 \leq 45$
$745 \times 1+985 \times 2+1150 \times 3+360 \times 4+315 \times 5+1294 \times 6+$
$552 \times 7+150 \times 8 \leq 10000$
$0 \times 1+0 \times 2+0 \times 3+1 \times 4+1 \times 5+0 \times 6+0 \times 7+0 \times 8 \leq 0$
$745 \times 1+985 \times 2+1150 \times 3+0 \times 4+0 \times 5+0 \times 6+0 \times 7+0$
$X 8 \leq 2000$

$\mathrm{X} 1, \mathrm{X} 2, \mathrm{X} 3, \mathrm{X} 4, \mathrm{X} 5, \mathrm{X} 6, \mathrm{X} 7, \mathrm{X} 8 \geq 0$

Modelo matemático de programación matemática, forma Matricial (Método Simplex):

MAXIMIZAR: $\mathrm{Z}=178750 \mathrm{X} 1+121960 \mathrm{X} 2+$ $209060 X 3+356901 X 4+250710 X 5+284762$

$\mathrm{X} 6+466728 \mathrm{X} 7+50000 \mathrm{X} 8+0 \mathrm{X} 9+0 \mathrm{X} 10+0$ $\mathrm{X} 11+0 \mathrm{X} 12+0 \mathrm{X} 13+0 \mathrm{X} 14+0 \mathrm{X} 15+0 \mathrm{X} 16+0$ $\mathrm{X} 17+0 \mathrm{X} 18+0 \mathrm{X} 19$

Sujeto a
$1 \mathrm{X} 1+1 \mathrm{X} 9=9$
$0 X 1+2 X 2+1 X 10=10$
$0 X 1+1 X 3+1 X 11=12$
$0 X 1+1 X 4+1 X 12=18$
$0 X 1+1 X 5+1 X 13=22$
$0 X 1+1 X 6+1 X 14=5$ 
$0 X 1+1 X 7+1 X 15=1$

$0 \mathrm{X} 1+1 \mathrm{X} 8+1 \mathrm{X} 16=45$

$745 X 1+985 X 2+1150 X 3+360 X 4+315 X 5+$ $1294 X 6+552 X 7+150 X 8+1 X 17=10000$

$0 \mathrm{X} 1+1 \mathrm{X} 4+1 \mathrm{X} 5+1 \mathrm{X} 18=0$

$745 X 1+985 X 2+1150 X 3+1 X 19=2000$

X1, X2, X3, X4, X5, X6, X7, X8, X9, X10, X11, $\mathrm{X} 12, \mathrm{X} 13, \mathrm{X} 14, \mathrm{X} 15, \mathrm{X} 16, \mathrm{X} 17, \mathrm{X} 18, \mathrm{X} 19 \geq 0$

\section{Resultados y discusión}

Tabla 3. Resultado de la primera interacción del método simplex

\begin{tabular}{|c|c|c|c|c|c|c|c|c|c|c|}
\hline Tabla 1 & & & 178750 & 121960 & 209060 & 356901 & 250710 & 284762 & 466728 & 50000 \\
\hline Base & $\mathbf{C b}$ & P0 & P1 & P2 & P3 & P4 & P5 & P6 & P7 & P8 \\
\hline P9 & 0 & 9 & 1 & 0 & 0 & 0 & 0 & 0 & 0 & 0 \\
\hline P10 & 0 & 10 & 0 & 2 & 0 & 0 & 0 & 0 & 0 & 0 \\
\hline P11 & 0 & 12 & 0 & 0 & 1 & 0 & 0 & 0 & 0 & 0 \\
\hline P12 & 0 & 18 & 0 & 0 & 0 & 1 & 0 & 0 & 0 & 0 \\
\hline $\mathrm{P} 13$ & 0 & 22 & 0 & 0 & 0 & 0 & 1 & 0 & 0 & 0 \\
\hline P14 & 0 & 5 & 0 & 0 & 0 & 0 & 0 & 1 & 0 & 0 \\
\hline P15 & 0 & 1 & 0 & 0 & 0 & 0 & 0 & 0 & 1 & 0 \\
\hline P16 & 0 & 45 & 0 & 0 & 0 & 0 & 0 & 0 & 0 & 1 \\
\hline P17 & 0 & 10000 & 745 & 985 & 1150 & 360 & 315 & 1294 & 552 & 150 \\
\hline P18 & 0 & 0 & 0 & 0 & 0 & 1 & 1 & 0 & 0 & 0 \\
\hline P19 & 0 & 2000 & 745 & 985 & 1150 & 0 & 0 & 0 & 0 & 0 \\
\hline $\mathbf{Z}$ & & 0 & -178750 & -121960 & -209060 & -356901 & -250710 & -284762 & -466728 & -50000 \\
\hline 0 & 0 & 0 & 0 & 0 & 0 & 0 & 0 & 0 & 0 & 0 \\
\hline P9 & P10 & P11 & P12 & P13 & P14 & P15 & P16 & P17 & P18 & P19 \\
\hline 1 & 0 & 0 & 0 & 0 & 0 & 0 & 0 & 0 & 0 & 0 \\
\hline 0 & 1 & 0 & 0 & 0 & 0 & 0 & 0 & 0 & 0 & 0 \\
\hline 0 & 0 & 1 & 0 & 0 & 0 & 0 & 0 & 0 & 0 & 0 \\
\hline
\end{tabular}

Para efectos de presentación, se mostrará a continuación los resultados generados en PHP-Simplex. Para llegar al resultado deseado, el modelo indica que deben pasar por ocho interacciones hasta llegar al resultado final. Se presentan las dos primeras interacciones y la séptima en este apartado, y de la tercera a la sexta se incluyen en los anexos de este documento (Anexos 1: interacción 3, Anexo 2: interacción 4, Anexo 3: interacción 5, Anexo 4: interacción 6, Anexo 5: interacción 7). 


\begin{tabular}{|c|c|c|c|c|c|c|c|c|c|c|}
\hline 0 & 0 & 0 & 1 & 0 & 0 & 0 & 0 & 0 & 0 & 0 \\
\hline 0 & 0 & 0 & 0 & 1 & 0 & 0 & 0 & 0 & 0 & 0 \\
\hline 0 & 0 & 0 & 0 & 0 & 1 & 0 & 0 & 0 & 0 & 0 \\
\hline 0 & 0 & 0 & 0 & 0 & 0 & 1 & 0 & 0 & 0 & 0 \\
\hline 0 & 0 & 0 & 0 & 0 & 0 & 0 & 1 & 0 & 0 & 0 \\
\hline 0 & 0 & 0 & 0 & 0 & 0 & 0 & 0 & 1 & 0 & 0 \\
\hline 0 & 0 & 0 & 0 & 0 & 0 & 0 & 0 & 0 & 1 & 0 \\
\hline 0 & 0 & 0 & 0 & 0 & 0 & 0 & 0 & 0 & 0 & 1 \\
\hline 0 & 0 & 0 & 0 & 0 & 0 & 0 & 0 & 0 & 0 & 0 \\
\hline
\end{tabular}

Fuente: Recolección de datos e información de medios publicitarios en Quito.

De acuerdo a la técnica del método simplex se indica la variable que «sale» de la base es P15 (resaltada en color verde) y a su vez la variable que «entra» es P7 que se encuentra en la misma columna.

Tabla 4. Resultado de la segunda interacción del método simplex

\begin{tabular}{|c|c|c|c|c|c|c|c|c|c|c|}
\hline Tabla 2 & & & 178750 & 121960 & 209060 & 356901 & 250710 & 284762 & 466728 & 50000 \\
\hline Base & $\mathbf{C b}$ & Po & P1 & $\mathbf{P 2}$ & $\mathbf{P 3}$ & P4 & P5 & P6 & P7 & P8 \\
\hline P9 & 0 & 9 & 1 & 0 & 0 & 0 & 0 & 0 & 0 & 0 \\
\hline P10 & 0 & 10 & 0 & 2 & 0 & 0 & 0 & 0 & 0 & 0 \\
\hline P11 & 0 & 12 & 0 & 0 & 1 & 0 & 0 & 0 & 0 & 0 \\
\hline P12 & 0 & 18 & 0 & 0 & 0 & 1 & 0 & 0 & 0 & 0 \\
\hline P13 & 0 & 22 & 0 & 0 & 0 & 0 & 1 & 0 & 0 & 0 \\
\hline P14 & 0 & 5 & 0 & 0 & 0 & 0 & 0 & 1 & 0 & 0 \\
\hline P7 & 466728 & 1 & 0 & 0 & 0 & 0 & 0 & 0 & 1 & 0 \\
\hline P16 & 0 & 45 & 0 & 0 & 0 & 0 & 0 & 0 & 0 & 1 \\
\hline P17 & 0 & 9448 & 745 & 985 & 1150 & 360 & 315 & 1294 & 0 & 150 \\
\hline P18 & 0 & 0 & 0 & 0 & 0 & 1 & 1 & 0 & 0 & 0 \\
\hline
\end{tabular}




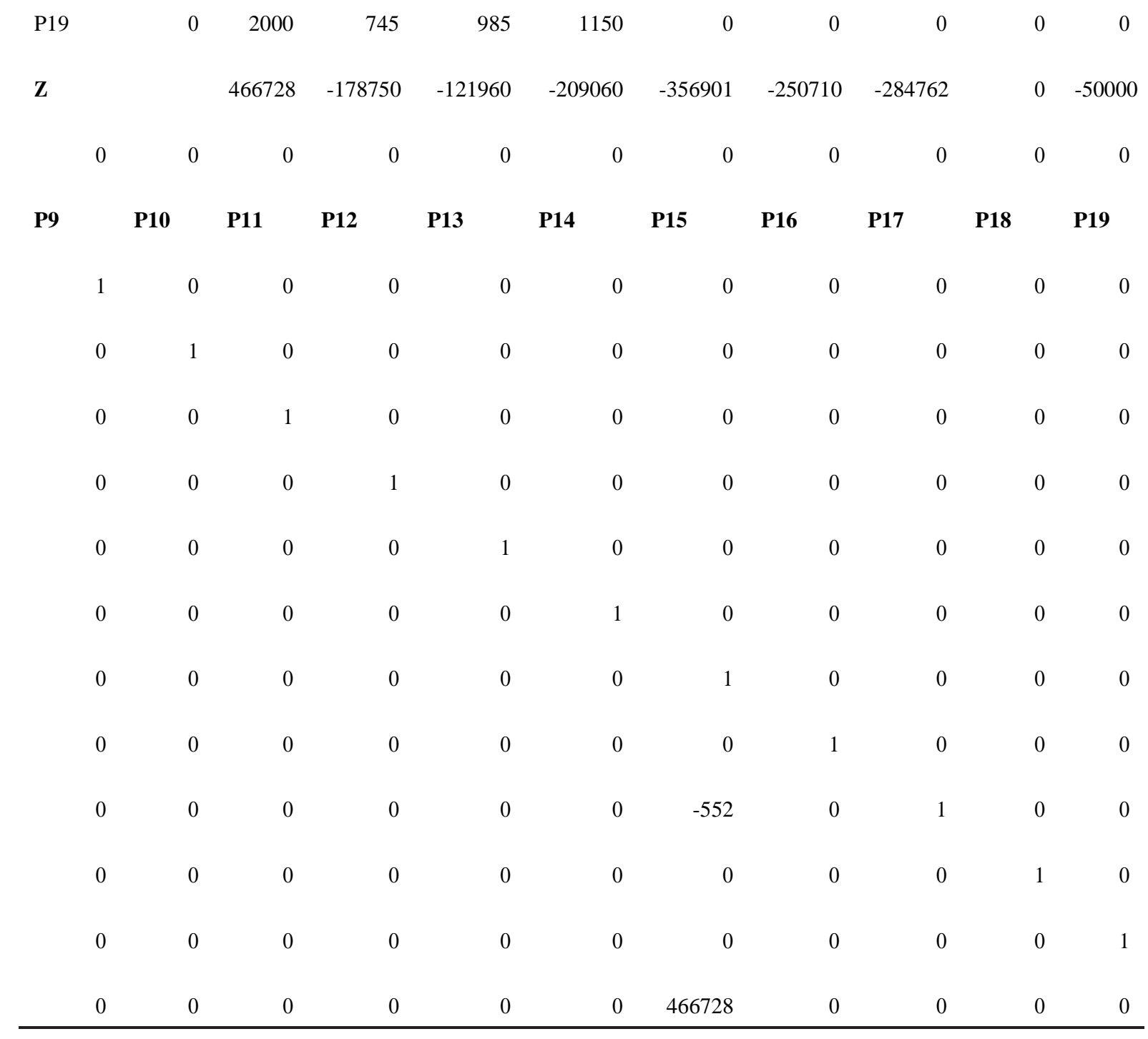

Fuente: Recolección de datos e información de medios publicitarios en Quito.

La interacción 2 del método simplex presenta que la variable que «sale» de la base de la matriz es P18 (resaltada en color verde) y a su vez la variable que «entra» en la matriz es P4 que se encuentra en la misma columna.

Tabla 5. Resultado de la séptima interacción del método simplex

\begin{tabular}{|c|c|c|c|c|c|c|c|c|c|c|}
\hline Tabla 8 & & & 178750 & 121960 & 209060 & 356901 & 250710 & 284762 & 466728 & 50000 \\
\hline Base & $\mathbf{C b}$ & P0 & P1 & P2 & P3 & P4 & P5 & P6 & P7 & P8 \\
\hline P9 & 0 & 6.315 & 0 & -1.322 & -1.543 & 0 & 0 & 0 & 0 & 0 \\
\hline P10 & 0 & 10 & 0 & 2 & 0 & 0 & 0 & 0 & 0 & 0 \\
\hline P11 & 0 & 12 & 0 & 0 & 1 & 0 & 0 & 0 & 0 & 0 \\
\hline
\end{tabular}




\begin{tabular}{|c|c|c|c|c|c|c|c|c|c|c|c|}
\hline P12 & & 0 & & 18 & 0 & 0 & 0 & 0 & -1 & 0 & 0 \\
\hline $\mathrm{P} 13$ & & 0 & & 22 & 0 & 0 & 0 & 0 & 1 & 0 & 0 \\
\hline P6 & & 284762 & & 0.539 & 0 & $-8,70 \mathrm{E}-04$ & 0 & 0 & $(-0.03)$ & 1 & 0 \\
\hline P7 & & 466728 & & 1 & 0 & 0 & 0 & 0 & 0 & 0 & 0 \\
\hline P14 & & 0 & & 4.460 & 0 & $8,70 \mathrm{E}-04$ & 0 & 0 & 0.034 & 0 & 0 \\
\hline P8 & & 50000 & & 45 & 0 & 0 & 0 & 0 & 0 & 0 & 1 \\
\hline P4 & & 356901 & & 0 & 0 & 0 & 0 & 1 & 1 & 0 & 0 \\
\hline P1 & & 178750 & & 2.684 & 1 & 1.322 & 1.543 & 0 & 0 & 0 & 0 \\
\hline $\mathbf{Z}$ & & & & .350198 & 0 & 1.143 & 6.686 & 0 & 9.628 & 0 & 0 \\
\hline 0 & 0 & 0 & 0 & 0 & 0 & 0 & 0 & & 0 & 0 & 0 \\
\hline P9 & P10 & P11 & P12 & P13 & P14 & P15 & P16 & & P17 & P18 & P19 \\
\hline 1 & 0 & 0 & 0 & 0 & 0 & 0 & 0 & & 0 & 0 & $(-0.001)$ \\
\hline 0 & 1 & 0 & 0 & 0 & 0 & 0 & 0 & & 0 & 0 & 0 \\
\hline 0 & 0 & 1 & 0 & 0 & 0 & 0 & 0 & & 0 & 0 & 0 \\
\hline 0 & 0 & 0 & 1 & 0 & 0 & 0 & 0 & & 0 & -1 & 0 \\
\hline 0 & 0 & 0 & 0 & 1 & 0 & 0 & 0 & & 0 & 0 & 0 \\
\hline 0 & 0 & 0 & 0 & 0 & 0 & $(-0.426)$ & $(-0.115)$ & & 0.0007 & $(-0.27)$ & $(-0.0007)$ \\
\hline 0 & 0 & 0 & 0 & 0 & 0 & 1 & 0 & & 0 & 0 & 0 \\
\hline 0 & 0 & 0 & 0 & 0 & 1 & 0.426 & 0.115 & & $(-0.0007)$ & 0.278 & 0.0007 \\
\hline 0 & 0 & 0 & 0 & 0 & 0 & 0 & 1 & & 0 & 0 & 0 \\
\hline 0 & 0 & 0 & 0 & 0 & 0 & 0 & 0 & & 0 & 1 & 0 \\
\hline 0 & 0 & 0 & 0 & 0 & 0 & 0 & 0 & & 0 & 0 & 0.001 \\
\hline 0 & 0 & 0 & 0 & 0 & 0 & 3.452 & 1.699 & & 2.200 & 2.776 & 1.986 \\
\hline
\end{tabular}

Fuente: Recolección de datos e información de medios publicitarios en Quito. 
Finalmente, en la interacción 7 se encuentra el resultado de maximizar el modelo matemático en el renglón $Z$ al final de la tabla.

La solución óptima para este modelo de programación lineal de ocho variables y once restricciones se muestra en el modelo matemático representado en su forma estándar, para luego ser registrado en forma de matriz. Para llegar al resultado final, el modelo requiere pasar por ocho interacciones; es decir, considera en cada una de las variables que «salen» del modelo y aquellas que «entran», representada en la tabla requerida del método simplex. La solución óptima requiere que los anuncios sean distribuidos en televisión matutina expresados en la variable $X 1$, con aproximadamente tres spots semanales. Del mismo modo el modelo requiere que con el presupuesto limitado de $\$ 10.000$ se genere actividad publicitaria en prensa entre los días lunes a sábado al menos en una ocasión, representado por la variable $X 6$; y, el domingo de acuerdo a la variable $X 7$. Finalmente, en el transcurso de la semana es indispensable que se utilicen las redes sociales como estrategia de medios publicitarios, siendo este el medio menos costoso y de cobertura en la actualidad. En este caso el modelo requiere de la totalidad de datos presentados inicialmente en la tabla 2 referente a medios de comunicación, costos y audiencia; es así que, los resultados son 45 anuncios como valor máximo en la semana; tomando en cuenta que, en el resto de medios publicitarios, al menos para este escenario, no deben ser utilizados.

La solución óptima es $Z=3350198.0036513$
$X 1=2.6845637583893$
$X 2=0$
$X 3=0$
$X 4=0$
$X 5=0$
$X 6=0.53941267387944$
$X 7=1$
$X 8=45$

En función de las restricciones, considerando costos, cobertura de audiencia y ciertas condiciones presentadas en el modelo, se espera que la cobertura de audiencia semanal utilizados en las variables $X 1$; $X 6$; $X 7$ y $X 8$ sea de 3.350.198 personas, con la combinación de todas las posibilidades que arroja el modelo.

\section{Conclusiones}

En Ecuador, la programación del marketing y la comunicación dentro de una estrategia competitiva y sostenible en el tiempo, todavía es una tarea pendiente. Apenas el 19,9\% de las organizaciones en este país han incorporado de manera decidida a esta ciencia, y a la utilización de medios online y offline para lograr mayor difusión de sus productos, servicio o soluciones en el mercado local o internacional; un $28,6 \%$ de empresas no utilizan para nada marketing dentro de sus modelos de negocio. Esto también está en concordancia con la generación de nuevas formas o canales de venta como las «plataformas de intercambio de productos» que para antes de la pandemia de covid-19 eran solamente usadas por el 5,8\% de las compañías comerciales, contra $61,6 \%$ que nunca habían pensado en esta forma de acercarse a los clientes. El mensaje publicitario y de comunicación también es un aspecto imprescindible para llegar a los consumidores, solamente un $8,3 \%$ de las empresas consideran importante la creación de contenidos y un $50,4 \%$ no le prestan atención a este importante detalle estratégico [28].

Para poder profesionalizar la toma de decisiones en estos aspectos, la programación lineal en cualquiera de sus técnicas, como es el caso del método simplex, propone la solución a problemas reales de las empresas. Haciendo uso de paquetes informáticos es posible simular múltiples variables y escenarios para la evaluación de estrategias que optimicen el costo y maximicen los beneficios, y, en este caso, generar la óptima cobertura de clientes. Son varias las ventajas que este tipo de modelos brinda, y que están al alcance de las empresas y sus miembros para aplicar técnicas de investigación con programación matemática en muchos campos empresariales como: inversiones, logística y operaciones, talento humano, investigación y marketing, economía, entre otros.

En la actualidad, con el uso de la tecnología, las empresas utilizan los medios de información como más crean conveniente de acuerdo a los fines necesarios. Por ejemplo, las redes sociales (que son un medio de comunicación masivo) llegan a una variada audiencia, y es un método muy explotado y aceptado por el público objetivo en los diferentes dispositivos tecnológicos actuales. 
Es importante resaltar que la técnica de programación matemática aplicada brinda un portafolio óptimo de asignaciones para lograr un resultado adecuado, permite evaluar los costos basados en la cobertura de audiencia requerida con un presupuesto limitado y fijo, identificando costos de oportunidad y rangos establecidos en los recursos. La facilidad de la formulación y adopción del software especializado, ayuda a desarrollar con precisión modelos lineales y no lineales; resaltando, además, que los profesionales involucrados en las empresas pueden generar la habilidad y destreza para abordar la resolución de problemas para maximizar las utilidades y minimizar los costos, así como también la asignación de tiempos a las actividades.

\section{Referencias}

[1] Alvarado, J. (2009). La Programación Lineal, Aplicación de las Pequeñas y Medianas Empresas. Reflexiones, 89-105. Obtenido de https://revistas.ucr.ac.cr/index.php/reflexiones/article/view/11511/10857

[2] AMA, American Marketing Association. (2008). Marketing Power. Obtenido de Marketing Definitions: http://www.marketingpower.com

[3] Anderson, D., Sweenwy, D., Williams, T., Camm, J., \& Matin, K. (2011). Métodos Cuantitativos para los Negocios. México: Cengage.

[4] Bermúdez, Y. (2011). Aplicaciones de Programación lineal, Entera y Mixta. Ingeniería industral, actualidad y nuevas tendencias, 85104.

[5] Bona, E., Borsato, D., Dos Santos Ferreira, R., \& Herera, R. (2000). Aplicativo para Otimização Empregando o Método Simplex Seqüencial. Acta Scientiarum, 1201-1206. Obtenido de http://repositorio.utfpr.edu. br:8080/jspui/handle/1/159

[6] Chandler, A. D. (1962). Strategy and Structure: Chapters in the History of the American Industrial Enterprise. Boston: MIT Press.

[7] Coll, V., \& Olga, B. (2006). Evaluación de la Eficiencia mediante Análisis Envolvente de Datos. Valencia: Universidad de Valencia.

[8] Correa, R., Begambre, O., \& Carrillo, J. (2010). Validación de un Algorítmo Hí- brido del Paso en el método Simplex y Topología de la Evolución Paramétrica. Dyna. Obtenido de https://www.redalyc.org/ pdf/496/49622372027.pdf

[9] De la Hoz, E., Vélez, J., \& López, L. (2017). Modelo de Programación Lineal Multiobjetivo para la Logística Inversa en el Sector Plástico de Polipropileno. Scielo Analytics -Información Tecnológica, 31-36. Obtenido de https://scielo.conicyt.cl/pdf/infotec/v28n5/ art05.pdf

[10] María, B. (2015). Optimización de Portafolio de Proyectos a través de la Aplicación de Programación Lineal y el CAPM. Ciencias estratégicas, 71-86. Obtenido de https://www. redalyc.org/pdf/1513/151353628005.pdf

[11] Mintzberg, H. (1993). El Proceso Estratégico: Conceptos y Casos. Barcelona: Prentice Hall.

[12] Molina, J., \& Morán, A. (2008). Viva la Publicidad Viva 3. Bogotá: Lemoine Editores.

[13] Montenegro, D. I. (2019). Investigación sobre la Competitividad en Empresas de Ecuador en la Era 4.0. Quito: IDE Business School.

[14] Montenegro, D. I., \& Calvache, M. (2016). EmotionShare: Estrategia y Marketing Disruptivo. Quito: Ecuador.

[15] Noguera, R., \& Posada, S. (2011). Programação Linear Aplicada à Formulação de Rações para Ruminantes. Revista CES Medicina Veterinaria y Zootecnia, 53-60. Obtenido de https://dialnet.unirioja.es/servlet/articulo? codigo $=3818632$

[16] Ortiz, V., \& Caicedo, A. (2014). Procedimiento para la Programación y Control de la Producción de una Pequeña Empresa de Calzado. Scientia Et Technica, 377384. Obtenido de https://www.redalyc.org/ pdf/849/84933912004.pdf

[17] Porter, M. (1985). Competitive Advantage: Creating and Sustaining Superior Performance. Boston: Free Press.

[18] Prieto, J., González, M., \& Arce, A. (2014). Aplicación de Programación Lineal en la Gestión de Carteras. Revista Científica Politécnica, 124-131. Obtenido de http://www. une.edu.py:83/fpunescientific/index.php/ fpunescientific/article/view/41

[19] Render, B., Stair, R., \& Hanna, M. (2012). Métodos Cuantitativos para los Negocios. México: Pearson. 
[20] Reyes, Y., Mula, J., Díaz, M., \& Gutierrez, E. (2017). Plan Maestro de producción basado en Métodos Cuantitativos para la Economía y Empresa, 147-168. Obtenido de https://www. econstor.eu/handle/10419/195385

[21] Schumpeter, J. A. (1911). The Theory of Economic Development. Boston: Harvard University Press.

[22] Soot, T. (2018). Matemáticas Aplicadas a los Negocios, las Ciencias Sociales y de la Vida. Toluca, México: Cengage Learning.
[23] Taha, H. (2011). Investigación de Operaciones. México: Pearson.

[24] Terrazas, R. (2012). Aplicación de la Programación Matemática a la Localización de Proyectos. Perspectivas, 71-94. Obtenido de http://www.scielo.org.bo/pdf/rp/n29/ n29a04.pdf

[25] Valls, J. (2014). Estrategias y Tácticas de Marketing. Barcelona: Profit.

[26] Wells, W., Moriarty, S., \& Burnett, J. (2007). Publicidad. Principios y Práctica. México: Pearson .

\section{Anexos}

Anexo 1: Interacción 3.

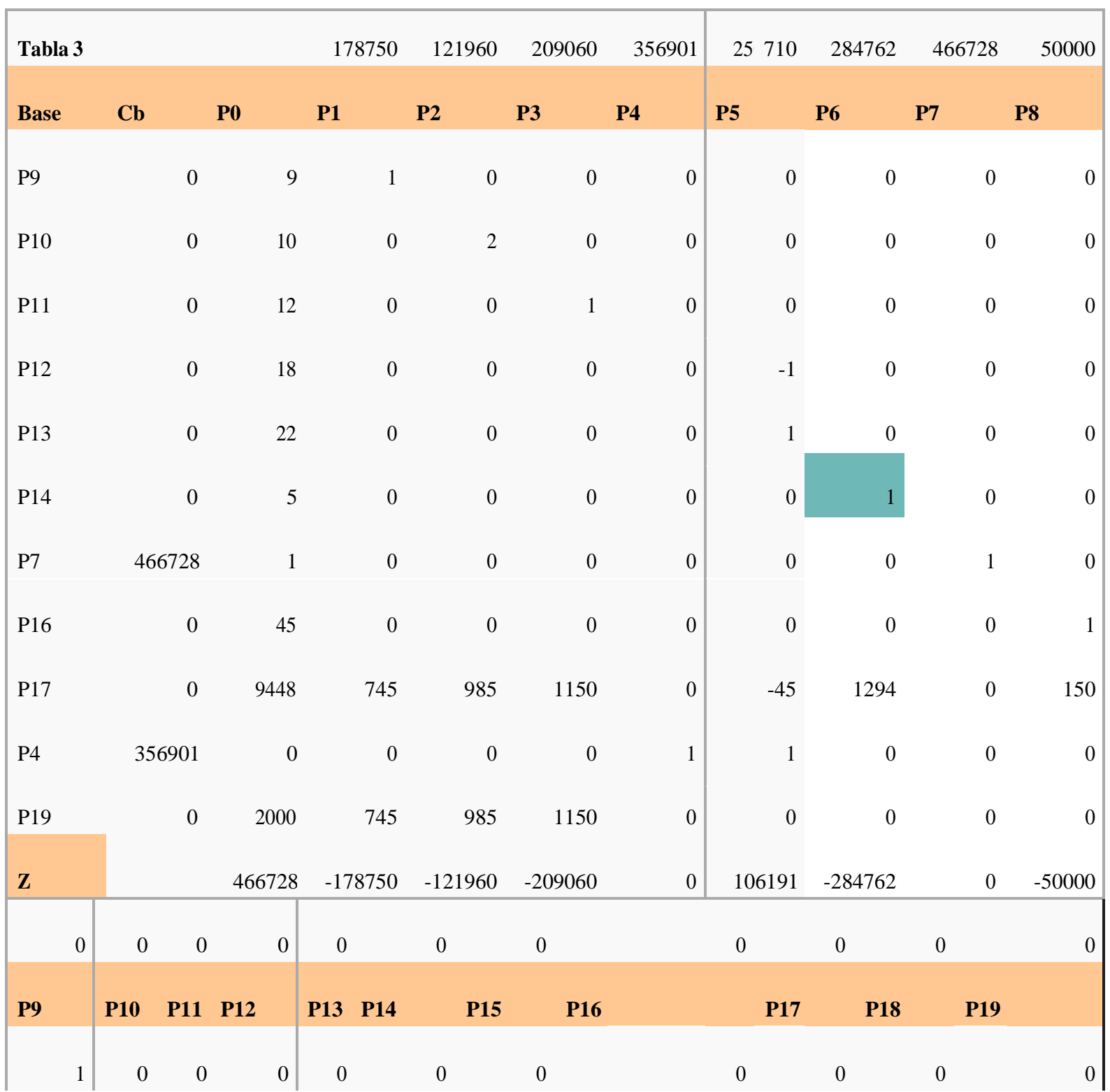




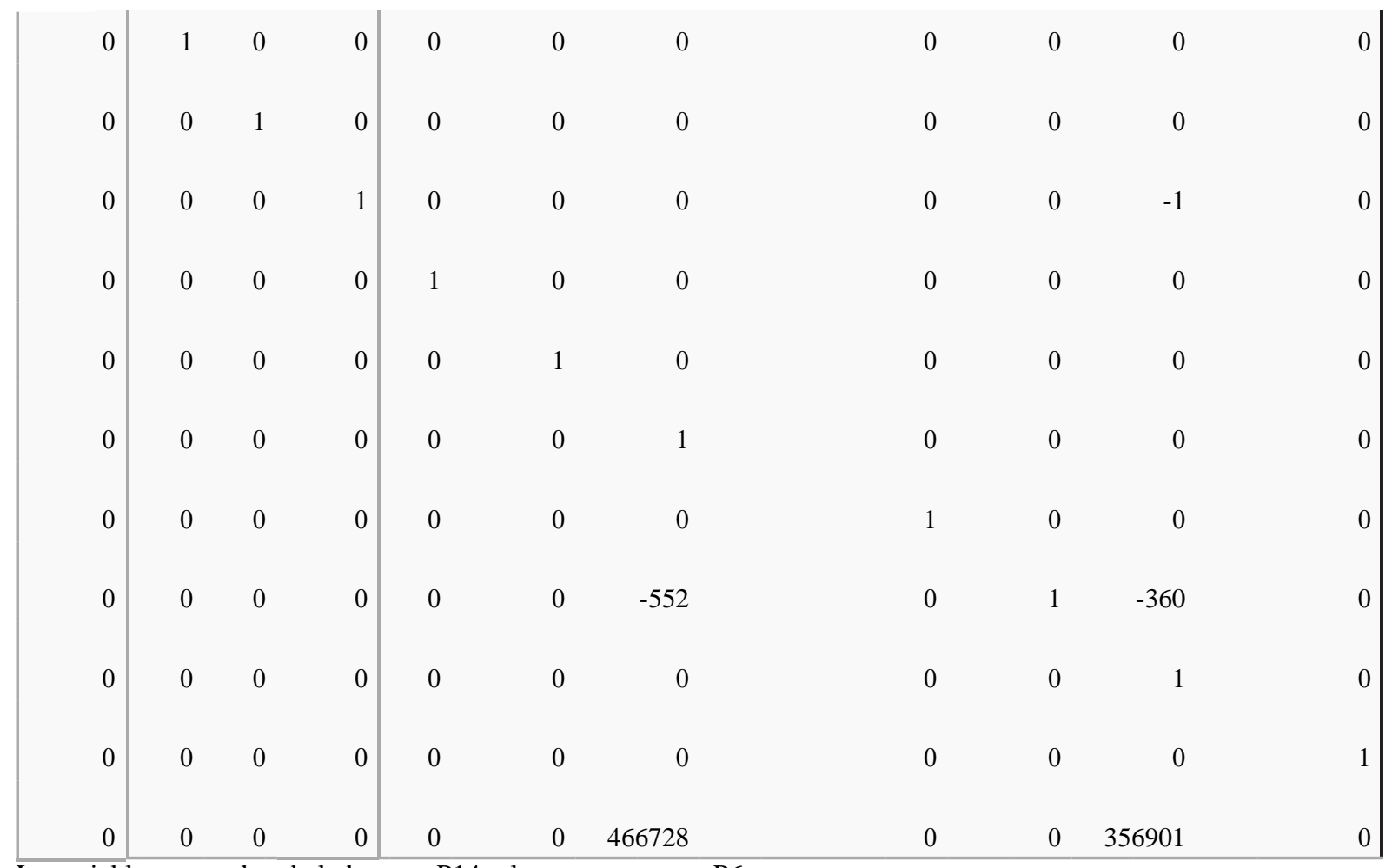

La variable que «sale» de la base es P14 y la que «entra» es P6.

Anexo 2: Interacción 4.

\begin{tabular}{|c|c|c|c|c|c|c|c|c|c|c|}
\hline Tabla 4 & & & 178750 & 121960 & 209060 & 356901 & 250710 & 284762 & 466728 & 50000 \\
\hline Base & $\mathbf{C b}$ & Po & P1 & P2 & P3 & P4 & P5 & P6 & P7 & P8 \\
\hline P9 & 0 & 9 & 1 & 0 & 0 & 0 & 0 & 0 & 0 & 0 \\
\hline P10 & 0 & 10 & 0 & 2 & 0 & 0 & 0 & 0 & 0 & 0 \\
\hline P11 & 0 & 12 & 0 & 0 & 1 & 0 & 0 & 0 & 0 & 0 \\
\hline P12 & 0 & 18 & 0 & 0 & 0 & 0 & -1 & 0 & 0 & 0 \\
\hline P13 & 0 & 22 & 0 & 0 & 0 & 0 & 1 & 0 & 0 & 0 \\
\hline P6 & 284762 & 5 & 0 & 0 & 0 & 0 & 0 & 1 & 0 & 0 \\
\hline P7 & 466728 & 1 & 0 & 0 & 0 & 0 & 0 & 0 & 1 & 0 \\
\hline P16 & 0 & 45 & 0 & 0 & 0 & 0 & 0 & 0 & 0 & 1 \\
\hline P17 & 0 & 2978 & 745 & 985 & 1150 & 0 & -45 & 0 & 0 & 150 \\
\hline P4 & 356901 & 0 & 0 & 0 & 0 & 1 & 1 & 0 & 0 & 0 \\
\hline P19 & 0 & 2000 & 745 & 985 & 1150 & 0 & 0 & 0 & 0 & 0 \\
\hline
\end{tabular}




\begin{tabular}{|l|r|r|r|r|r|r|r|r|r|}
\hline $\mathbf{Z}$ & 1890538 & -178750 & -121960 & -209060 & 0 & 106191 & 0 & 0 & -50000 \\
\hline
\end{tabular}

\begin{tabular}{|c|c|c|c|c|c|c|c|c|c|c|}
\hline 0 & 0 & 0 & 0 & 0 & 0 & 0 & 0 & 0 & 0 & 0 \\
\hline P9 & P10 & P11 & P12 & P13 & P14 & P15 & P16 & P17 & P18 & P19 \\
\hline 1 & 0 & 0 & 0 & 0 & 0 & 0 & 0 & 0 & 0 & 0 \\
\hline 0 & 1 & 0 & 0 & 0 & 0 & 0 & 0 & 0 & 0 & 0 \\
\hline 0 & 0 & 1 & 0 & 0 & 0 & 0 & 0 & 0 & 0 & 0 \\
\hline 0 & 0 & 0 & 1 & 0 & 0 & 0 & 0 & 0 & -1 & 0 \\
\hline 0 & 0 & 0 & 0 & 1 & 0 & 0 & 0 & 0 & 0 & 0 \\
\hline 0 & 0 & 0 & 0 & 0 & 1 & 0 & 0 & 0 & 0 & 0 \\
\hline 0 & 0 & 0 & 0 & 0 & 0 & 1 & 0 & 0 & 0 & 0 \\
\hline 0 & 0 & 0 & 0 & 0 & 0 & 0 & 1 & 0 & 0 & 0 \\
\hline 0 & 0 & 0 & 0 & 0 & -1294 & -552 & 0 & 1 & -360 & 0 \\
\hline 0 & 0 & 0 & 0 & 0 & 0 & 0 & 0 & 0 & 1 & 0 \\
\hline 0 & 0 & 0 & 0 & 0 & 0 & 0 & 0 & 0 & 0 & 1 \\
\hline 0 & 0 & 0 & 0 & 0 & 284762 & 466728 & 0 & 0 & 356901 & 0 \\
\hline
\end{tabular}

La variable que «sale» de la base es P19 y la que «entra»es P3.

\section{Anexo 3: Interacción 5.}

\begin{tabular}{|c|c|c|c|c|c|c|c|c|c|c|}
\hline Tabla 5 & & & 178750 & 121960 & 209060 & 356901 & 250710 & 284762 & 466728 & 50000 \\
\hline Base & $\mathbf{C b}$ & P0 & P1 & P2 & P3 & P4 & P5 & P6 & P7 & P8 \\
\hline P9 & 0 & 9 & 1 & 0 & 0 & 0 & 0 & 0 & 0 & 0 \\
\hline P10 & 0 & 10 & 0 & 2 & 0 & 0 & 0 & 0 & 0 & 0 \\
\hline P11 & 0 & 1.026 & $(-0.64)$ & $(-0.85)$ & 0 & 0 & 0 & 0 & 0 & 0 \\
\hline P12 & 0 & 18 & 0 & 0 & 0 & 0 & -1 & 0 & 0 & 0 \\
\hline P13 & 0 & 22 & 0 & 0 & 0 & 0 & 1 & 0 & 0 & 0 \\
\hline P6 & 2847 & 5 & 0 & 0 & 0 & 0 & 0 & 1 & 0 & 0 \\
\hline P7 & 4667 & 1 & 0 & 0 & 0 & 0 & 0 & 0 & 1 & 0 \\
\hline
\end{tabular}




\begin{tabular}{|c|c|c|c|c|c|c|c|c|c|c|}
\hline P16 & \multicolumn{2}{|c|}{0} & 45 & 0 & \multicolumn{2}{|l|}{0} & 0 & 0 & 0 & 1 \\
\hline P17 & \multicolumn{2}{|c|}{0} & 978 & 0 & \multicolumn{2}{|c|}{$-1,14 \mathrm{E}+00$} & 0 & -45 & 0 & 150 \\
\hline $\mathrm{P} 4$ & \multicolumn{2}{|c|}{3569} & 0 & 0 & \multicolumn{2}{|l|}{0} & 1 & 1 & 0 & 0 \\
\hline P3 & \multirow{2}{*}{\multicolumn{2}{|c|}{2090}} & 1.739 & 0.647 & \multicolumn{2}{|c|}{0.856} & 0 & 0 & 0 & 0 \\
\hline $\mathbf{Z}$ & & & 2.254 & $(-433)$ & \multicolumn{2}{|c|}{5.710} & 0 & 106191 & 0 & -50000 \\
\hline 0 & 0 & 0 & 0 & 0 & 0 & 0 & 0 & 0 & 0 & 0 \\
\hline P9 & P10 & P11 & P12 & P13 & P14 & P15 & P16 & P17 & P18 & P19 \\
\hline 1 & 0 & 0 & 0 & 0 & 0 & 0 & 0 & 0 & 0 & 0 \\
\hline 0 & 1 & 0 & 0 & 0 & 0 & 0 & 0 & 0 & 0 & 0 \\
\hline 0 & 0 & 1 & 0 & 0 & 0 & 0 & 0 & 0 & 0 & $(-0.0008)$ \\
\hline 0 & 0 & 0 & 1 & 0 & 0 & 0 & 0 & 0 & -1 & 0 \\
\hline 0 & 0 & 0 & 0 & 1 & 0 & 0 & 0 & 0 & 0 & 0 \\
\hline 0 & 0 & 0 & 0 & 0 & 1 & 0 & 0 & 0 & 0 & 0 \\
\hline 0 & 0 & 0 & 0 & 0 & 0 & 1 & 0 & 0 & 0 & 0 \\
\hline 0 & 0 & 0 & 0 & 0 & 0 & 0 & 1 & 0 & 0 & 0 \\
\hline 0 & 0 & 0 & 0 & 0 & -1294 & -552 & 0 & 1 & -360 & -1 \\
\hline 0 & 0 & 0 & 0 & 0 & 0 & 0 & 0 & 0 & 1 & 0 \\
\hline 0 & 0 & 0 & 0 & 0 & 0 & 0 & 0 & 0 & 0 & 0.0008 \\
\hline 0 & 0 & 0 & 0 & 0 & 284762 & 466728 & 0 & 0 & 356901 & 1.817 \\
\hline
\end{tabular}

La variable que «sale» de la base es P17 y la que «entra» es P8.

\section{Anexo 4: Interacción 6.}

\begin{tabular}{|cccccc|cccccc|}
\hline Tabla 6 & & & 178750 & 121960 & 209060 & 356901 & 250710 & 284762 & 466728 & 50000 \\
Base & Cb & P0 & P1 & P2 & P3 & $\mathbf{P 4}$ & $\mathbf{P 5}$ & $\mathbf{P 6}$ & $\mathbf{P 7}$ & $\mathbf{P 8}$ \\
P9 & 0 & 9 & 1 & 0 & 0 & 0 & 0 & 0 & 0 & 0 \\
P10 & 0 & 10 & 0 & 2 & 0 & 0 & 0 & 0 & 0 & 0 \\
P11 & 0 & 1.026 & $(-0.64)$ & $(-0.856)$ & 0 & 0 & 0 & 0 & 0 & 0 \\
P12 & 0 & 18 & 0 & 0 & 0 & 0 & -1 & 0 & 0 & 0
\end{tabular}




\begin{tabular}{|c|c|c|c|c|c|c|c|c|c|c|c|}
\hline P13 & \multicolumn{2}{|c|}{0} & 22 & 0 & 0 & 0 & 0 & & & 0 & 0 \\
\hline P6 & \multicolumn{2}{|c|}{284762} & 5 & 0 & 0 & 0 & 0 & & & 1 & 0 \\
\hline P7 & \multicolumn{2}{|c|}{466728} & 1 & 0 & 0 & 0 & 0 & & & 0 & 0 \\
\hline P16 & \multicolumn{2}{|c|}{0} & 38.48 & 0 & $7,58 \mathrm{E}-03$ & 0 & 0 & & & 0 & 0 \\
\hline P8 & \multicolumn{2}{|c|}{50000} & 6.52 & 0 & $\begin{array}{c}-7,58 \mathrm{E}- \\
03\end{array}$ & 0 & 0 & \multicolumn{2}{|c|}{-0.3} & 0 & 1 \\
\hline $\mathrm{P} 4$ & \multicolumn{2}{|c|}{356901} & 0 & 0 & 0 & 0 & 1 & & & 0 & 0 \\
\hline P3 & \multicolumn{2}{|c|}{209060} & 1.739 & 0.647 & 0.856 & 1 & 0 & & & 0 & 0 \\
\hline $\mathbf{Z}$ & & & 2.580 & $(-433)$ & 5.710 & 0 & 0 & & & 0 & 0 \\
\hline 0 & 0 & 0 & 0 & 0 & 0 & 0 & & 0 & 0 & 0 & 0 \\
\hline P9 & P10 & P11 & P12 & P13 & P14 & P15 & & P16 & P17 & P18 & P19 \\
\hline 1 & 0 & 0 & 0 & 0 & 0 & 0 & & 0 & 0 & 0 & 0 \\
\hline 0 & 1 & 0 & 0 & 0 & 0 & 0 & & 0 & 0 & 0 & 0 \\
\hline 0 & 0 & 1 & 0 & 0 & 0 & 0 & & 0 & 0 & 0 & $(-0.0008)$ \\
\hline 0 & 0 & 0 & 1 & 0 & 0 & 0 & & 0 & 0 & -1 & 0 \\
\hline 0 & 0 & 0 & 0 & 1 & 0 & 0 & & 0 & 0 & 0 & 0 \\
\hline 0 & 0 & 0 & 0 & 0 & 1 & 0 & & 0 & 0 & 0 & 0 \\
\hline 0 & 0 & 0 & 0 & 0 & 0 & 1 & & 0 & 0 & 0 & 0 \\
\hline 0 & 0 & 0 & 0 & 0 & 8.626 & 3.68 & & 1 & $(-0.006)$ & 2.4 & 0.006 \\
\hline 0 & 0 & 0 & 0 & 0 & $(-862)$ & -3.68 & & 0 & 0.006 & -2.4 & $(-0.006)$ \\
\hline 0 & 0 & 0 & 0 & 0 & 0 & 0 & & 0 & 0 & 1 & 0 \\
\hline 0 & 0 & 0 & 0 & 0 & 0 & 0 & & 0 & 0 & 0 & 0.0008 \\
\hline 0 & 0 & 0 & 0 & 0 & $(-146)$ & 282728 & & 0 & 3.333 & 236901 & $(-151)$ \\
\hline
\end{tabular}

La variable que «sale» de la base es P16 y la que «entra» es P14. 
Anexo 5: Interacción 7.

\begin{tabular}{|c|c|c|c|c|c|c|c|c|c|c|}
\hline Tabla 7 & & & 178750 & 121960 & 209060 & 356901 & 250710 & 284762 & 466728 & 50000 \\
\hline Base & $\mathbf{C b}$ & Po & P1 & P2 & P3 & P4 & P5 & P6 & P7 & P8 \\
\hline P9 & 0 & 9 & 1 & 0 & 0 & 0 & 0 & 0 & 0 & 0 \\
\hline P10 & 0 & 10 & 0 & 2 & 0 & 0 & 0 & 0 & 0 & 0 \\
\hline P11 & 0 & 1.026 & $(-0.64)$ & $(-0.85)$ & 0 & 0 & 0 & 0 & 0 & 0 \\
\hline P12 & 0 & 18 & 0 & 0 & 0 & 0 & -1 & 0 & 0 & 0 \\
\hline $\mathrm{P} 13$ & 0 & 22 & 0 & 0 & 0 & 0 & 1 & 0 & 0 & 0 \\
\hline P6 & 284762 & 0.539 & 0 & $-8,79 \mathrm{E}-04$ & 0 & 0 & $(-0.03)$ & 1 & 0 & 0 \\
\hline P7 & 466728 & 1 & 0 & 0 & 0 & 0 & 0 & 0 & 1 & 0 \\
\hline P14 & 0 & 4.460 & 0 & 8,79E-04 & 0 & 0 & 0.034 & 0 & 0 & 0 \\
\hline P8 & 50000 & 45 & 0 & 0 & 0 & 0 & 0 & 0 & 0 & 1 \\
\hline P4 & 356901 & 0 & 0 & 0 & 0 & 1 & 1 & 0 & 0 & 0 \\
\hline P3 & 209060 & 1.739 & 0.647 & 0.85652173913043 & 1 & 0 & 0 & 0 & 0 & 0 \\
\hline $\mathbf{Z}$ & & 3.233 & $(-433)$ & 5.710 & 0 & 0 & 9.628 & 0 & 0 & 0 \\
\hline
\end{tabular}

\begin{tabular}{|c|c|c|c|c|c|c|c|c|c|c|}
\hline 0 & 0 & 0 & 0 & 0 & 0 & 0 & 0 & 0 & 0 & 0 \\
\hline P9 & P10 & P11 & P12 & P13 & P14 & P15 & P16 & P17 & P18 & P19 \\
\hline 1 & 0 & 0 & 0 & 0 & 0 & 0 & 0 & 0 & 0 & 0 \\
\hline 0 & 1 & 0 & 0 & 0 & 0 & 0 & 0 & 0 & 0 & 0 \\
\hline 0 & 0 & 1 & 0 & 0 & 0 & 0 & 0 & 0 & 0 & $(-0.0008)$ \\
\hline 0 & 0 & 0 & 1 & 0 & 0 & 0 & 0 & 0 & -1 & 0 \\
\hline 0 & 0 & 0 & 0 & 1 & 0 & 0 & 0 & 0 & 0 & 0 \\
\hline 0 & 0 & 0 & 0 & 0 & 0 & $(-0.42)$ & $(-0.11)$ & 0.0007 & $(-0.27)$ & $(-0.0007)$ \\
\hline 0 & 0 & 0 & 0 & 0 & 0 & 1 & 0 & 0 & 0 & 0 \\
\hline 0 & 0 & 0 & 0 & 0 & 1 & 0.426 & 0.115 & $(-0.0007)$ & 0.278 & 0.0007 \\
\hline 0 & 0 & 0 & 0 & 0 & 0 & 0 & 1 & 0 & 0 & 0 \\
\hline
\end{tabular}




\begin{tabular}{|c|c|c|c|c|c|c|c|c|c|c|}
\hline 0 & 0 & 0 & 0 & 0 & 0 & 0 & 0 & 0 & 1 & 0 \\
\hline 0 & 0 & 0 & 0 & 0 & 0 & 0 & 0 & 0 & 0 & 0.0008 \\
\hline 0 & 0 & 0 & 0 & 0 & 0 & 3.452 & 1.699 & 2.200 & 2.776 & -3.827 \\
\hline
\end{tabular}

La variable que «sale» de la base es P3 y la que «entra» es P1. 